\title{
Choice of optimum heights for registration of ionospheric response onto earthquakes
}

\author{
Valerii Krasnov", Ivan Gotur, Yurii Kuleshov and Sergei Cherny \\ Mozhaisky Military Space Academy, 197198, Saint Petersburg, Zhdanovskaya,13, Russia
}

\begin{abstract}
To investigate the dependence of ionospheric disturbances on height we used model calculations, and the data of seismic and ionospheric observations during the Tohoku-Oki earthquake. High-altitude dependences of "portraits" of ionospheric disturbances are calculated for a case of influence of a seismic P-wave onto the ionosphere. We compared the "portraits" of ionospheric disturbances with the "portraits" of the seismic recording. The correlation coefficient of the recordings for the height of 100 $\mathrm{km}$ was about 0.81 , for $130 \mathrm{~km}-0.85$, for $160 \mathrm{~km}-0.77$, for $180 \mathrm{~km}-0.76$, for $200 \mathrm{~km}-0.7$, for $230 \mathrm{~km}$ 0.54 and for $250 \mathrm{~km}-0.41$. At the same time the maximum of F2-layer was at the height about $250 \mathrm{~km}$. Thus, the height of a maximum of F2-layer was not optimum for registration of ionospheric disturbances due to the earthquake. It was preferable to carry out measurements of the ionospheric disturbances at the heights below $200 \mathrm{~km}$. The profile of amplitude of the ionospheric disturbance had no sharply expressed maximum at the height of a maximum of F2-layer. Therefore it is problematic to use the approach of the thin layer for interpretation of TEC disturbances.
\end{abstract}

\section{Introduction}

Development of Global Navigation Satellite Systems has opened new opportunities to investigate ionospheric disturbances based on the space-time variations of total electron content (TEC). For example, the paper ensemble appears in the literature after strong earthquake, which describe results of influence of movement of a terrestrial surface or a tsunami upon the ionosphere. It is offered to use this kind of observations in tsunami warning systems, and also during monitoring of earth (sea) surface motion in region, which is difficult of access (for example, $[1,2]$ ). The principal disadvantage of this method is integrated character of a measurable value of TEC along trajectory of a radio wave (for example, [3]). However, the assumption is accepted that the main contribution to TEC variations appears around the height of the maximum of ionosphere ionization (F2 layer). Integrated character of TEC does not allow defining precisely height of observable ionospheric disturbances and their coordinate. There are attempts to define the conditions at which ionospheric disturbances can be most precisely located and to the greatest degree are similar to a seismic signal. For example [4], it is recommended to carry out the measurements at a satellite elevation angle lower than $40^{\circ}$. The purpose of our work, by means of model calculations and the observation data of the Tohoku-Oki earthquake, to investigate dependence of the form of ionospheric disturbances on height and to define the optimum heights for observation of the ionospheric response onto the earthquake.

\section{Models used for the purposes of calculations}

To do investigation we used the data of seismic stations of the Czech Republic, the data of ionosonde, located in Pruhonice, and the data of ionospheric Doppler radio sounding. The detailed description of the network of observation points is presented in [5], and Fig.1 shows location of the network.

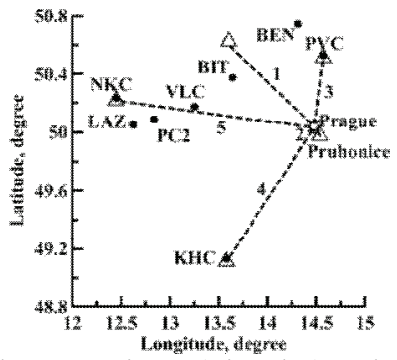

Fig. 1. Position of seismic stations (circles), Doppler transmitters (triangles) and Doppler shift receiver (Prague). Dashed lines show the five paths of Doppler radio sounding of the ionosphere.

*Corresponding author: vmkrasnov@yandex.ru 
The epicentre of a strong earthquake $(M=9.0)$ was at a distances of $\sim 9000 \mathrm{~km}$ from a point of observation. Seismographs have registered arrival P, S, SS and Rayleigh waves. As an example, in this work we consider a case of influence of a P wave onto the ionosphere. The model of vertical motion of earth surface is developed on the basis of seismic data and in detail described in [6]. The basic oscillations of the earth surface motion $\mathrm{S}(\mathrm{t})$ under action of $\mathrm{P}$ wave occurred in the frequencies range of $0.0195 \mathrm{~Hz}$ (the period $-51.2 \mathrm{~s}$ ) and $0.039 \mathrm{~Hz}$ (the period $25.6 \mathrm{~s}$, Fig.2).
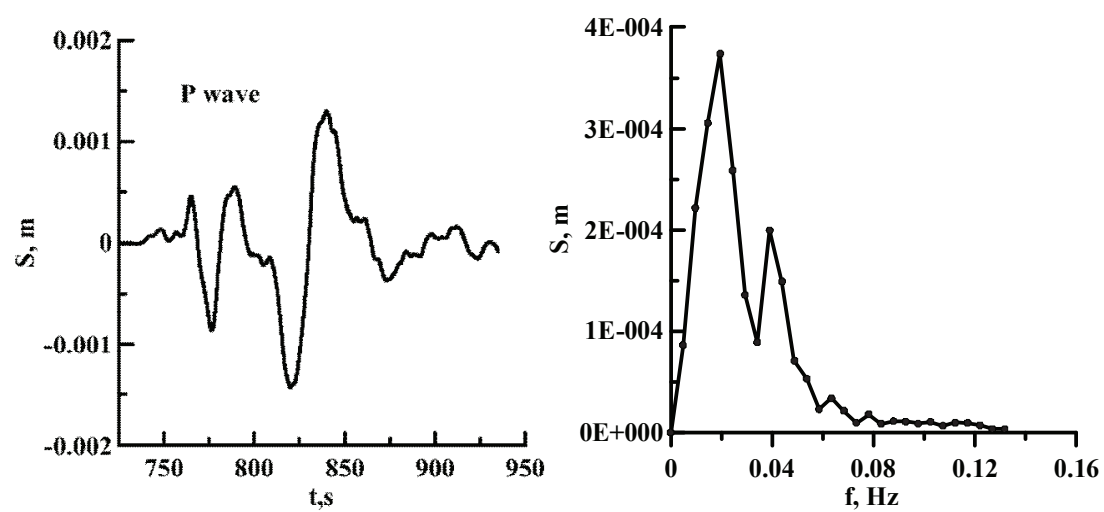

Fig. 2. "Portrait" of motion of the earth surface as a function of time for $P$ wave (a) and its spectrum (b).

The model of radiation of an infrasound by ground motion is developed on the basis of the Rayleigh integral

$$
p(t)=\frac{\rho_{0}}{2 \pi} \int \frac{a[t-2 / c)}{2} d S
$$

where $p(t)$ is the derived pressure history at any arbitrary point in space, $t$ is time, $\rho_{0}$ is the air density at the surface, $L$ is the slant range from the surface element to the position (observation point), $a(t-L / c)$ is the retarded acceleration normal to the earth surface, and $d S$ is the surface element of the emitting source.

The knowledge of the height of the observation points allows us to resolve task of choice of the circle radius size to calculate the Rayleigh integral. For this purpose it is necessary to define maximal horizontal distance from the observation point up to the surface element, from which the acoustic ray can propagate to the observation points. For example, the earth's surface with radius of $240 \mathrm{~km}$ forms an acoustic field at a height of $215 \mathrm{~km}$ during the TohokuOki earthquake [6].

The Rayleigh integral (1) assumes linear propagation of acoustic wave through a homogeneous medium without attenuation. However, the Rayleigh integral may be used and for calculation of infrasound field in the inhomogeneous atmosphere. In particular, there is the model for a point source, which allows calculation of the acoustic field taking into account atmospheric inhomogeneity, nonlinear effects, absorption and divergence of the wave front because of longrange acoustic wave propagation [7]. Krasnov et al. [6] have developed an algorithm, which can be used to adapt this model and the Rayleigh integral for the calculation of infrasonic fields caused by an earthquake at heights of the ionosphere.

To describe the evolution of an acoustic signal along acoustic ray we used the formula [7]

$$
V=\frac{b}{\varepsilon c \rho_{0}} \frac{\partial}{\partial t}(\ln U)
$$

where $V, \rho_{0}$, are the velocity and density of the fluid, respectively; $c$ is a sound speed,

$$
U\left(z, t_{p}\right)=\frac{1}{2 q \sqrt{\pi}} \int_{-\infty}^{+\infty} U_{i n}\left(0, t^{\prime}\right) \exp \left\{\frac{-\left(t-t^{\prime}\right)^{2}}{4 q^{2}}\right\} d t^{\prime}
$$

where $\varepsilon=\frac{\gamma+1}{2} ; b=\left(\zeta+\frac{4}{3} \eta\right)+\left(\frac{1}{c_{v}}-\frac{1}{c_{p}}\right) \chi, \zeta$ and $\eta$ bulk and shear viscosities, $\chi$ is the thermal conductivity, $\gamma$ is the ratio of specific heats $\mathrm{cp} / \mathrm{cv}, t_{p}=t+\int \frac{f(z)}{c} d z, \mathrm{t}$ is running time, $f(z)=\left(1-\frac{3 a+d}{2 \rho_{0} c^{2}} \frac{\partial c}{\partial z}-\frac{3 a}{2 \rho_{0}^{2} c} \frac{\partial \rho_{0}}{\partial z}\right)$, $\mathrm{a}=\zeta+\frac{4}{\mathrm{a}} \eta, d=\left(\frac{1}{c_{v}}-\frac{1}{c_{p}}\right) \chi, q^{2}=\int \frac{b}{2 \rho_{0} c^{3}} d z$ is response time of "atmospheric filter". 
Within altitudes below $\sim 100 \mathrm{~km}$ (region $\mathrm{D}$ and the lower part of region $\mathrm{E}$ of the ionosphere), both ions and electrons move together with neutral particles; therefore, it is possible to assume [8]

$$
N(t) \approx N_{0}\left(1 \pm \frac{\gamma-1}{2} \frac{v(s)}{c}\right)^{2 /(\gamma-1)}
$$

where $N_{0}$ is the background concentration of electrons in the ionosphere and $N$ is the concentration of electrons under the effect of the acoustic wave.

Above $130 \mathrm{~km}$, the ionospheric plasma is considered magnetized, and the following formula [9] is required to estimate the influence of the acoustic perturbation on the formation of the disturbance of electron concentration:

$$
\frac{\partial N}{\partial t}=-\left[N_{0}\left(\frac{v}{L} \sin ^{2} \Theta+\frac{\partial v}{\partial t} \cos ^{2} \Theta\right)+v \frac{\partial N_{0}}{d z} \cos \varphi \cos \Theta\right]
$$

where $\Theta$ is the angle between direction of the geomagnetic field and the acoustic ray trajectory, and $\varphi$ is the angle between direction of the geomagnetic field and $z$ axis, $l$ is coordinate along acoustic ray, $L$ is the slant range from the surface element to the position (observation point).

We calculate the Doppler shift of the radio wave as the time derivative of the transmitted phase which is given by

$$
f_{d}=-\frac{f}{c_{L}} \frac{d}{d t} \int_{s} n \cos \alpha d s
$$

where $\mathrm{f}$ is the carrier frequency of the radio sounder, $c_{L}$ is the speed of light, $\alpha$ is the angle between trajectory of radio ray and $z$ axis, $s$ is the path of radio ray from transmitter to receiver, $n$ is the reflection coefficient. We did not neglect by the magnetic field.

Validity of the models was confirmed by comparison of the calculations and data of the Doppler radio sounding of the ionosphere for five paths (Fig.1). The correlation coefficient between calculated and experimental records for seismic waves: P, S, SS and the first Rayleigh waves was at the range from 0.68 to 0.9. As an example, Fig. 3 shows the calculated and experimental Doppler shifts for the P wave and cross-correlation coefficients $(\mathrm{k})$ between the calculated and experimental curves.
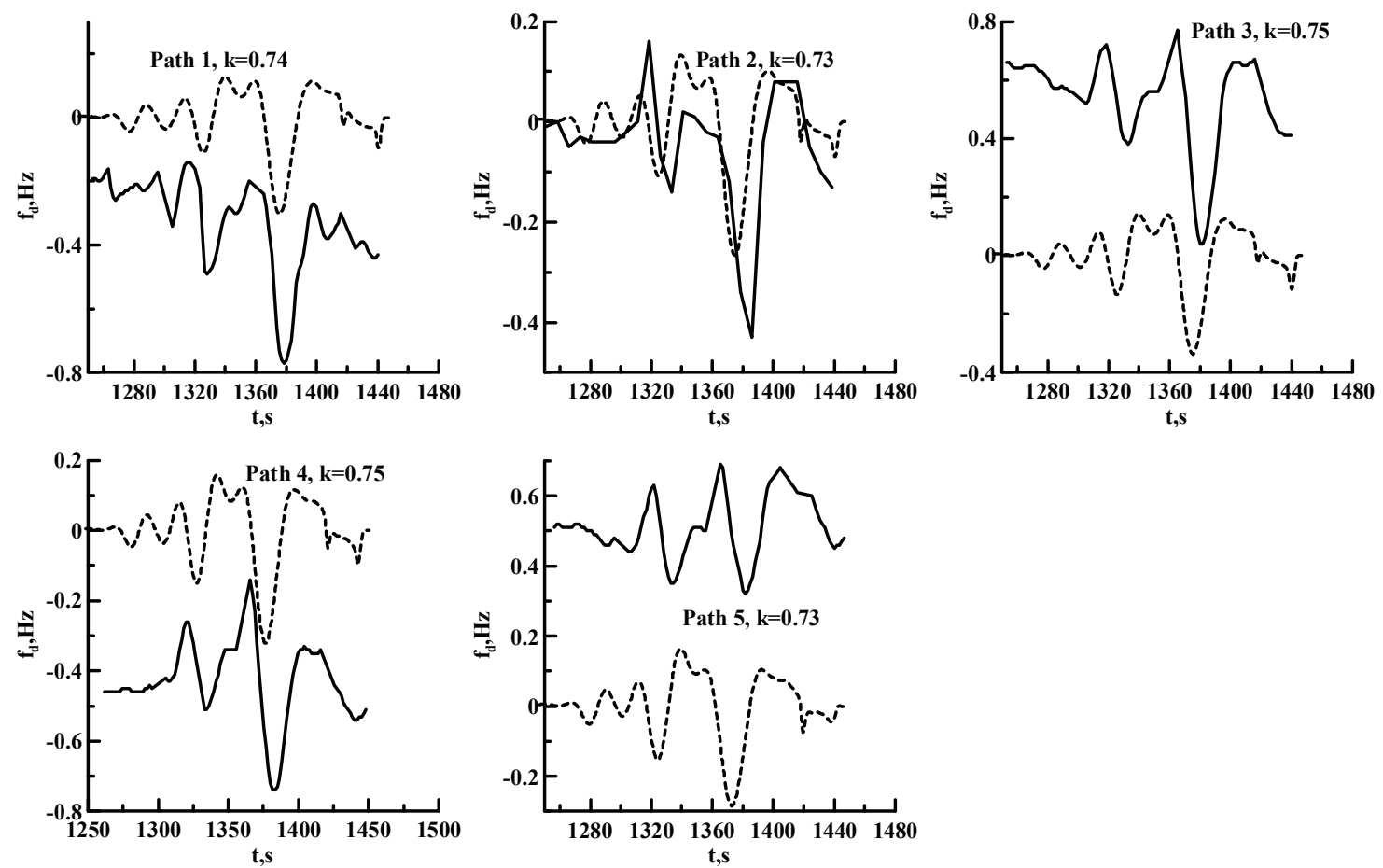

Fig.3. Experimental (solid lines) and calculated (dotted lines) forms of Doppler frequency for five radio traces of the ionospheric sounding during the $\mathrm{P}$ seismic wave propagation. Zero time is time of the earthquake.

\section{Dependence of the ionospheric disturbances on height}

The ionospheric electron density profile (Fig. 4) was estimated from the ionogram measured by the digital ionosonde located in Pruhonice (Fig.1) 


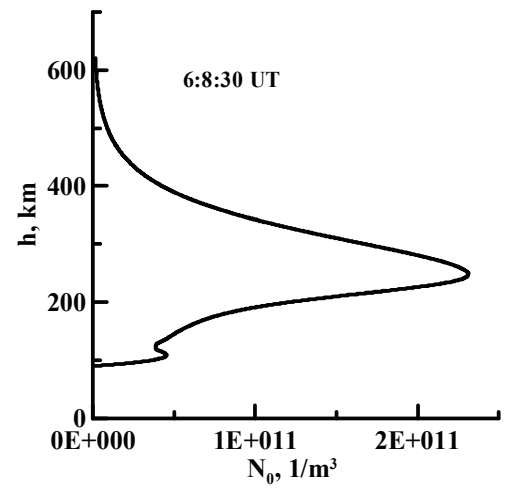

Fig.4. The ionospheric electron density profile.

The profile $\mathrm{N}(\mathrm{z})$ begins with height of $\sim 90 \mathrm{~km}$, and the maximum is located at the height of $250 \mathrm{~km}$.

First of all it is interesting to compare amplitudes and forms of the earth surface motion and the motion of hydrodynamical particles at different heights of the atmosphere. Fig. 5 shows the calculated motions of hydrodynamical particles under action of the acoustic wave at different altitudes.
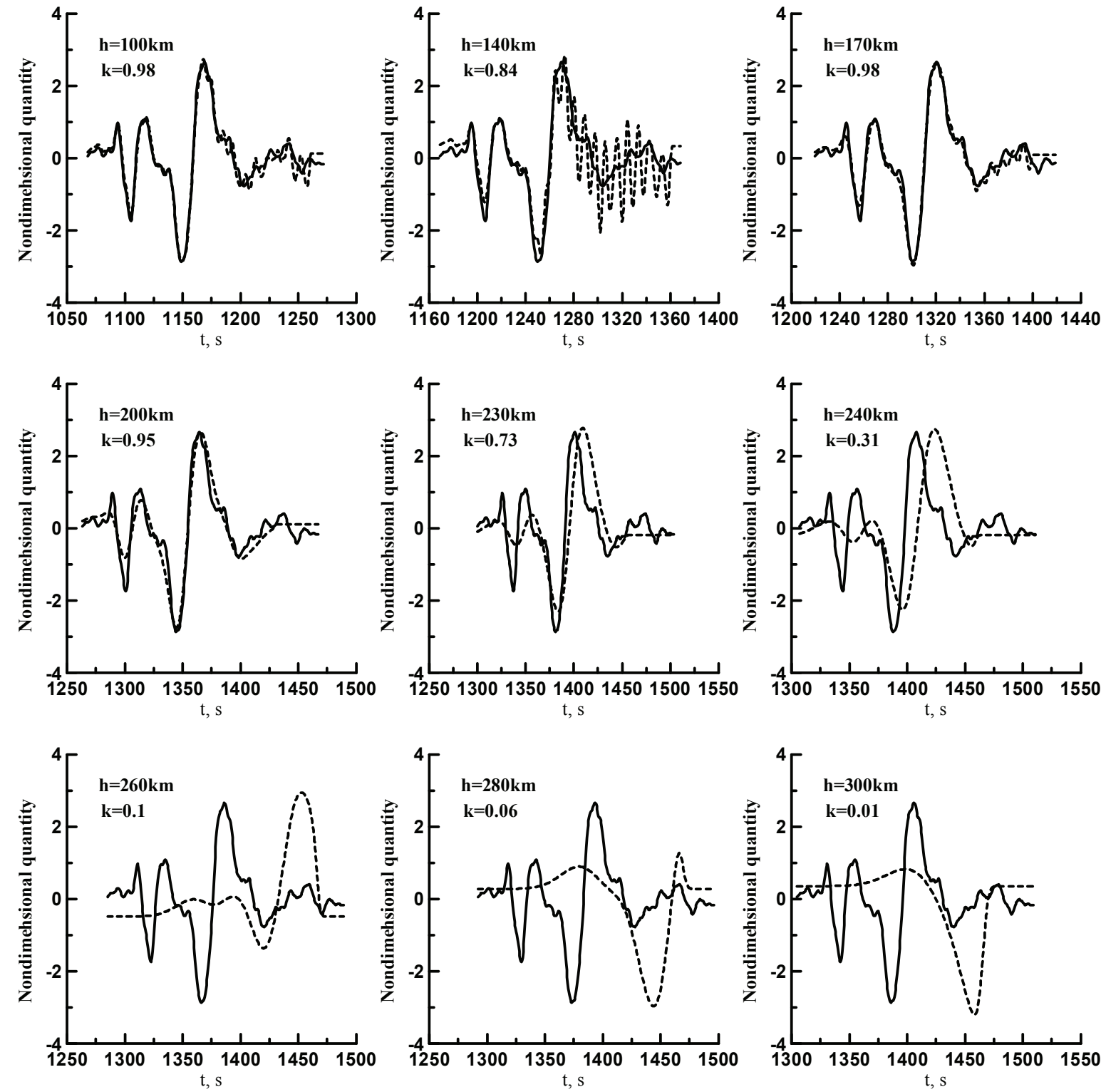

Fig. 5. The comparison of forms of the earth surface motion (solid line) and motions of hydrodynamical particles (dotted line) at different heights. $\mathrm{K}$ is a correlation coefficient. 
Additional high-frequency oscillations of motions of hydrodynamical particles at heights of 100 and $140 \mathrm{~km}$ have arisen because of an interference of the acoustic waves radiated by different areas of the earth surface. These high-frequency oscillations are destroyed by the nonlinear processes during its propagation upward to the altitude of $170 \mathrm{~km}$. As a whole, good similarity of motion of the earth surface and hydrodynamical particles is observed up to height of $230 \mathrm{~km}$ $(\mathrm{k} \geq 0.73)$. Then nonlinear processes strongly change the form of motion of hydrodynamical particles. In practice, seismographs observe a vertical speed $\left(V_{z}=\frac{d s}{d z}\right)$ of an earth surface motion, and radiophysical methods observe disturbances of electron density. Fig. 6 shows the results of comparison of a seismogram for P waves and calculated disturbances of electron density at different heights.
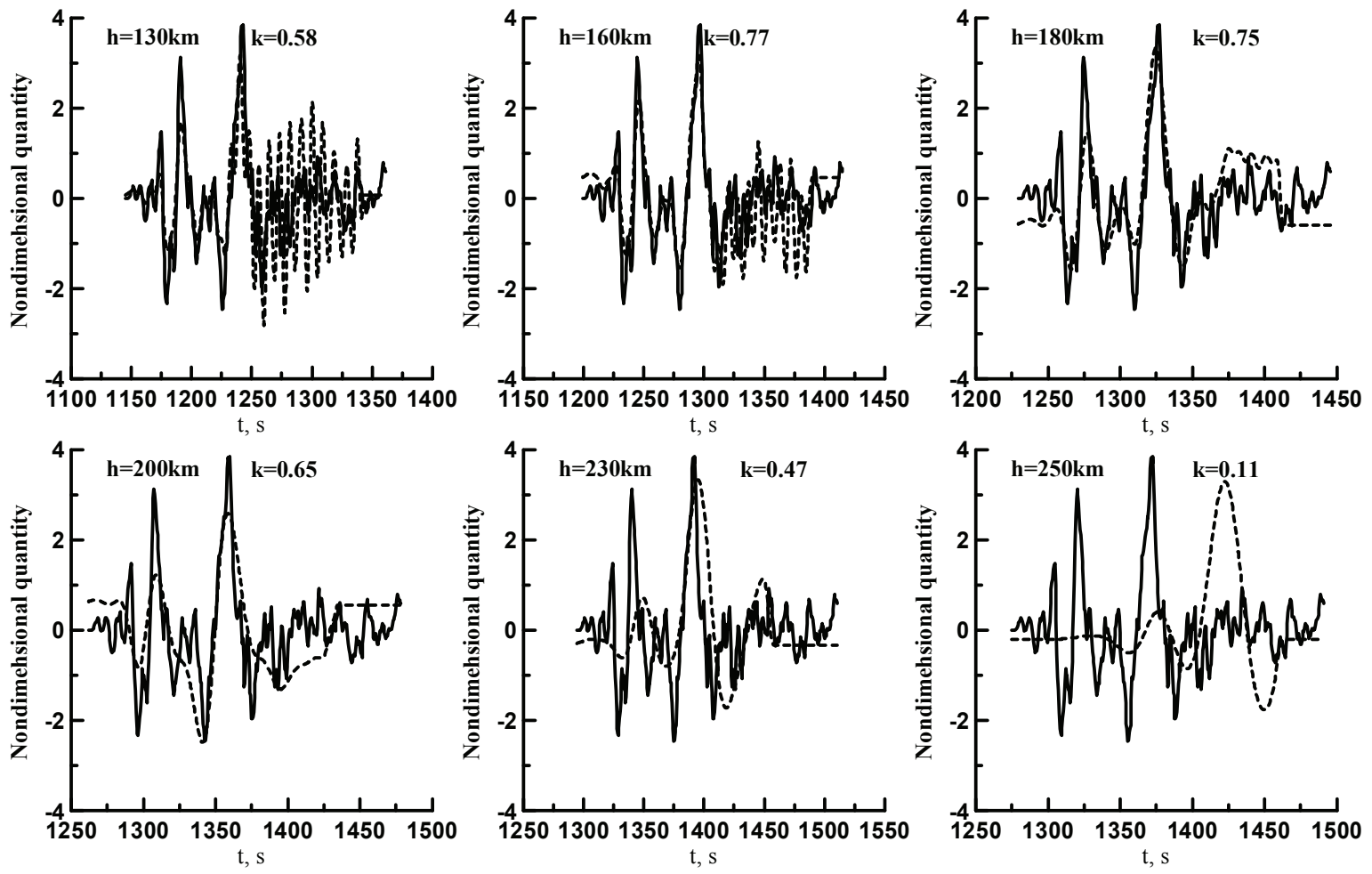

Fig. 6. The comparison of forms of $\mathrm{P}$ wave (solid line) and disturbances of electron density at different heights (dotted line). K is a correlation coefficient.

Good similarity of oscillations of vertical velocity of the earth surface and disturbances of electron density is observed up to height of $200 \mathrm{~km}(\mathrm{k} \geq 0.7)$. It means that heights of the ionosphere below $200 \mathrm{~km}$ are most preferable to observe the ionospheric response to the influence of $\mathrm{P}$-wave. It is obvious, that for registration of these disturbances it is necessary to use oblique trajectory of radio wave propagation from satellites to a receiver. This conclusion is in agreed with the results of experiment by Rolland et al. [4].

Disturbances of electron density arise in the ionosphere due to propagation upwards of an infrasonic wave. As a result, the disturbances of electron density are shifted from each other in time (Fig. 7).

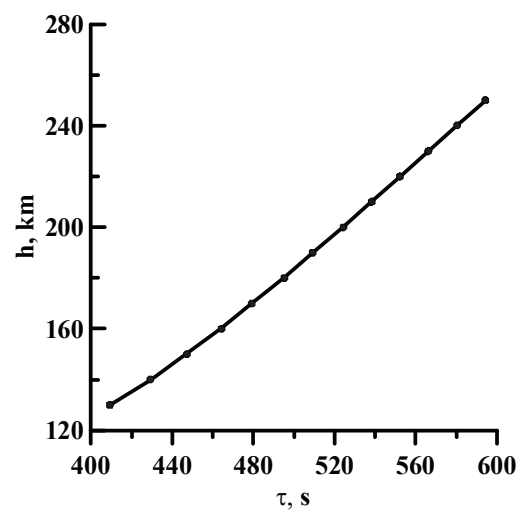

Fig. 7. Time delay of influence of the P-wave on formation of ionospheric disturbances at different height. 
It leads to summation of the disturbances in different phases of motion, when we measure TEC along a trajectory of radio wave, and it leads to additional distinction of a seismogram and TEC. It is obvious, that the distinction of forms of oscillations will increase with decrease of wave-length of the disturbances.

It is assumed that the main contribution to TEC variations appears around the height of the maximum of ionosphere ionization (F2 layer). This allows one to consider the ionosphere as a thin layer located at the height $\mathrm{H}$. TEC then represents a point of intersection of a line of sight with this thin layer. Let us consider dependence of deviation amplitude of the ionospheric disturbances on height for our case. Fig. 8 shows the calculation results of dependence of root-mean-square deviations $\left(\sigma_{N}\right)$ of ionospheric disturbance on height.
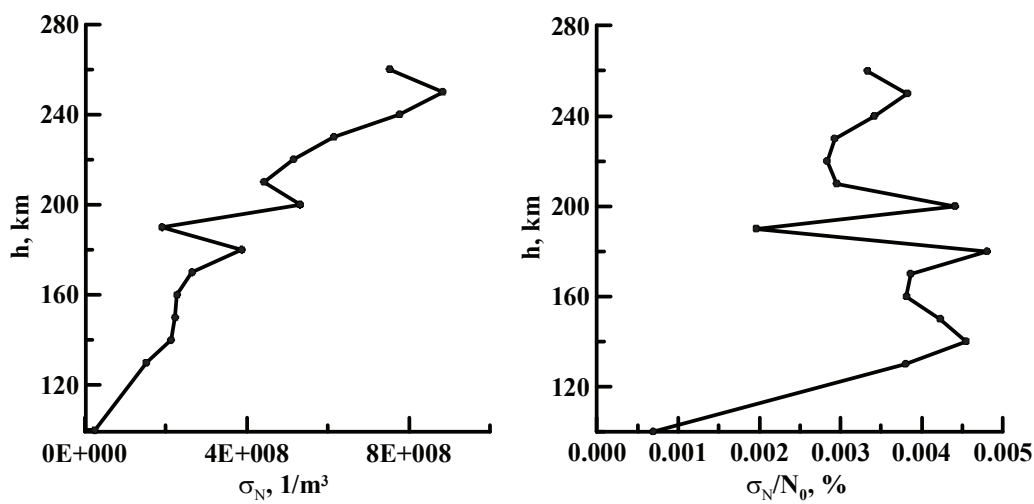

Fig. 8. The calculation results of dependence of $\sigma_{\mathbb{N}}$ on height: a) on absolute value; b) in percentage in relation to background value of electron density.

Small amplitude of the disturbances at the fixed height explains small values of TEC during earthquakes. There is a small maximum of disturbance around the maximum of the ionospheric F2 layer. Thus, it is a problem to use the approach of a thin layer for interpretation of the TEC.

Spectra of S, SS and Rayleigh waves of the Tohoku earthquake contain more low-frequency oscillations. Therefore nonlinear effects will essentially influence them at greater heights. Accordingly the upper border of optimum registration of the ionospheric response to these waves will be at higher height, than for P-wave.

\section{Conclusion}

We have considered influence of a P-wave on the ionosphere during the Tohoku-Oki earthquake. To do research we used the model, which allows calculation of the acoustic field taking into account atmospheric inhomogeneity, nonlinear effects, absorption and divergence of the wave front because of long-range acoustic wave propagation. Highaltitude variations of "portraits" of ionospheric disturbances were determined. We compared the "portraits" of ionospheric disturbances with the "portraits" of the seismic recording. The correlation coefficient of the recordings for the height of $100 \mathrm{~km}$ was about 0.81 , for $130 \mathrm{~km}-0.85$, for $160 \mathrm{~km}-0.77$, for $180 \mathrm{~km}-0.76$, for $200 \mathrm{~km}-0.7$, for 230 $\mathrm{km}-0.54$ and for $250 \mathrm{~km}-0.41$. At the same time the maximum of F2-layer was at the height about $250 \mathrm{~km}$. Thus, the height of a maximum of F2-layer was not optimum for registration of ionospheric disturbances due to the earthquake. As a result we defined the border of heights $(<200 \mathrm{~km})$ up to which the temporal oscillation of ionospheric disturbances are similar to the form of seismic records. The knowledge of this border and the characteristics of a directional radiation pattern of acoustic wave can help to choose the optimum trajectory of radiowave propagation from a satellite to a receiver to measure TEC. The profile of amplitude of the ionospheric disturbance had no explicitly expressed maximum at the height of a maximum of F2-layer. Therefore it is problematic to use the approach of the thin layer for interpretation of TEC disturbances.

\section{References}

1. P. Coïsson, P. Lognonné, D. Walwer, L. M. Rolland, Earth Space Sci. 2 (5), 125 (2015).

2. A. Komjathy, D. A. Galvan, P. Stephens, M. D. Butala, V. Akopian, B. Wilson, O. Verkhoglyadova, A. J. Mannucci, and M. Hickey, Earth Planets Space, 64, 1287 (2012).

3. E. Astafyeva, L. Rolland, P. Lognonné, K. Khelfi, T. Yahagi, J. Geophys. Res.: Space Phys. 118 (9), 5942 (2013).

4. L. M. Rolland, P. Lognonné and H. Munekane, J. Geophys. Res. 116, A05320 (2011).

5. J. Chum, F. Hruska, J. Zednik and J. Lastovicka, J. Geophys. Res. 117, A08319 (2012).

6. V. M. Krasnov, Ya.V. Drobzheva, J.,F. Chum, J. Atmos.Sol.-Terr.Phys. 135, 12 (2015).

7. Ya. Drobzheva, V. Krasnov, J. Atmos. Sol.-Terr.Phys. 65 (3), 369 (2003). 
8. V. M. Krasnov and Yu. V. Kuleshov, Acous. Phys. 60 (1), 19 (2014).

9. V.M. Krasnov, G.M. Pelenitcin, O.E. Ryaskov, N.M. Salikhov, Vestn. Acad. Sci. Kazakh USR, 8,44 (1991). 